\title{
You need to see what you want to understand-ultrastructure helps to uncover the mysteries of early life
}

\author{
Peter Nick
}

(C) Springer-Verlag Wien 2013

The early evolution of eukaryotes has remained a realm of mystery and surprise. The qualitative leap from the prokaryotes to cells, that bear complex and dynamic compartments, is still one of the hardest nuts to be cracked for evolutionary theory. The concept of "symbiogenesis", postulated by Mereshkowski at the beginning of the last century and much later solidified through molecular evidence by Margulis emerged as central concept to understand, how qualitative and complex traits could be acquired by endosymbiosis. However, Mereshkowski and Margulis left as a burden to evolutionary biology the insight that the conventional model of phylogenetic trees has to be replaced by more reticulate concepts of descendence. This working program is still far from being completed-we have got only glimpses into the complexity of early eukaryotic life. Since the underlying mechanism, endosymbiosis, is cellular, the approach to distangle this complexity must be cell biological in the first place - this remains valid even in the age of molecular genetics. This means that we need ultrastructural research to understand the early evolution of eukaryotes. Three contributions in the current issue perpetuate a long history of this journal on ultrastructural insights on early eukaryotes.

Diatoms are curious creatures that seem to lack flagellae. However, they have meanwhile been assigned to the heterokont Chromista, a group that is characterized by two flagella of different length and comprises quite diverse life forms such as Brown Algae or Oomycetes. The work by Idei et al. (2013) in the current issue investigates the ultrastructure of the flagellum for the sperm of the diatoms Thalassiosira lacustris and Melosira moniliformis, based on high-quality serial sections using conventional chemical fixation and contrasting. They show that the sperms are uniflagellate, with no trace of a second basal body (unexpected for heterokonts) and carry an axoneme with a $9+0$ microtubule configuration, and a basal body with only a doublet structure. They report further

P. Nick $(\bowtie)$

Karlsruhe, Germany

e-mail: Peter.nick@kit.edu exotic features of the microtubular cytoskeleton in these diatoms, and also specific differences between the two taxa that complement classical studies on the cytoskeleton in the heterokont Chromista (Hardham 1987, Andersen 1991).

Eukaryotic cells arose from endosymbiotic events of bacteria that were later transformed into mitochondria and plastids. The Rickettsiales are bacteria that are obligate endosymbionts and have attracted considerable interest as closest recent relatives of mitochondria, but also as clinically relevant pathogens. The work by Kawafune et al. (2013) in the current issue reports now on a unique case, where Ricketsialike bacteria live as endosymbionts in the unicellular green alga Carteria cerasiformis. By high-pressure freezing and freeze substitution and transmission electron microscopy analysis they can now reveal, in a series of astounding images, numerous cellular details of endosymbiosis and can discern the membrane structure of the endosymbiont which allows to assign them to the genus Rickettsia and to exclude the genus Orientia as source of the endosymbiont.

Red algae have been generated by secondary endosymbiosis, where heterokont organisms merged with a fungal ancestor. No wonder that cell division in red algae bears some peculiarities that are not only interesting for their exotism, but also because they are relevant to understand the cellular evolution of early eukaryotes. The work by Yagisawa et al. (2013) in the current issue extends previous work on mitotic inheritance of different organelles such as mitochondria and plastids (Maruyama and Kuroiwa 2010) or the endoplasmic reticulum (Yagisawa et al. 2012) to the Golgi body in the red alga model Cyanidioschyon merolae. By immunofluorescence they detect either one or two Golgi bodies per cell that are inherited together with the spindle poles, a phenomenon that has also been observed for organelles in Toxoplasma gondii and Trypanosoma brucei, important human pathogens that also have evolved from repeated cycles of secondary endosymbiosis. The finding that various organelles use the microtubules for inheritance, is consistent with a model, where the duplicated centrioles that are directly 
linked to microtubules as transport system, have acted as general division vehicle to ensure symmetric partitioning of cellular organelles. Evolutionary comparisons of early eukaryotic mitosis (reviewed in Schmit and Nick 2008) shows that also the link of the chromosomes with the spindle was originally indirect suggesting that the mechanism revealed in $C$. merolae represents something like a living fossile of cell division in ancestral eukaryotes.

\section{References}

Andersen RA (1991) The cytoskeleton of chromophyte algae. Protoplasma 164:143-159

Hardham AR (1987) Microtubules and the flagellar apparatus in zoospores and cysts of the fungus Phytophthora cinnamomi. Protoplasma 137:109-124
Idei M, Osada K, Sato S, Nakayama T, Nagumo T, Mann DG (2013) Sperm ultrastructure in the diatoms Melosira and Thalassiosira and the significance of the $9+0$ configuration. Protoplasma. doi:10.1007/ s00709-012-0465-8

Kawafune K, Sato M, Toyooka K, Nozaki H (2013) Ultrastructure of the rickettsial endosymbiont "MIDORIKO" in the green alga Carteria cerasiformis as revealed by high-pressure freezing and freezesubstitution fixation. Protoplasma. doi:10.1007/s00709-012-0469-4

Maruyama S, Kuroiwa T (2010) Division of cell nuclei, mitochondria, plastids and microbodies mediated by mitotic spindle poles in the primitive red alga Cyanidioschyzon merolae. Protoplasma 241:63-74

Schmit AC, Nick P (2008) Microtubules and the evolution of mitosis. Plant Cell Monogr 143:233-266

Yagisawa F, Fujiwara T, Kuroiwa H, Nishida K, Imoto Y, Kuroiwa T (2012) Mitotic inheritance of endoplasmic reticulum in the primitive red alga Cyanidioschyzon merolae. Protoplasma 249:1129-1135

Yagisawa F, Fujiwara T, Ohnuma M, Kuroiwa H, Nishida K, Yoshida Y, Kuroiwa T (2013) Golgi inheritance in the primitive red alga Cyanidioschyzon merolae. Protoplasma. doi:10.1007/s00709012-0467-6 